\title{
RESISTENCIA DEL $P$. FALCIPARUM A DROGAS EN COLOMBIA 1961-1986
}

\author{
SYLVIA BLAIR TRUJILLO*
}

\section{INTRODUCCION}

Diferentes informes $(24,27,3,4,8)$ refieren que la resistencia de los Plasmodium a las drogas antimaláricas, principalmente del $P$. falciparum, es uno de los "problemas técnicos" que han contribuido a agravar la situación de la malaria tanto en Colombia como en el mundo, y, por lo tanto, también se le atribuye papel en la dificultad para la erradicación del paludismo (12, $31)$.

Repetidamente se hace referencia a la resistencia del $P$. falciparum a la cloroquina en Colombia con expresiones como: "la distribución geográfica de esa resistencia corresponde a la distribución del $P$. falciparum" (31); el informe de 1961 de 2 casos de resistencia anticipó lo que se convertiría en un problema de rápida expansión (16); en Colombia todas las áreas maláricas excepto la Costa Pacífica presentan resistencia a la cloroquina por $P$. falciparum (2); en la mayoría de las áreas maláricas existe resistencia a las principales drogas como cloroquina y amodiaquina $\mathrm{y}$ tanto in vivo como in vitro se ha demostrado que entre el $70 \mathrm{y}$ el $90 \%$ de las infecciones son resistentes a la amodiaquina y un $60 \%$ a la cloroquina y a la quinina (10); las cepas resistentes se encuentran diseminadas por todo el territorio; la mayor parte son resistentes I y II, pero es evidente un aumento de la resistencia III (3).

Las referencias sobre estudios de resistencia in vivo $\mathrm{o}$ in vitro son muy escasas, pero son citadas una $\mathrm{y}$ otra vez, creando una cadena sin fin compuesta por muy pocos eslabones.

\section{MATERIAL Y METODOS}

Tratando de resolver interrogantes sobre este tema se hizo una revisión de la literatura colombiana al respecto, tomando como fuente inicial la existente en la biblioteca de la Facultad de Medicina de la Universidad de Antioquia, que a su vez consultó la red nacional de Bibliotecas Médicas. Luego se tomaron las referencias citadas en cada uno de los artículos anteriores y, en una búsqueda en cadena, se logró reunir y revisar 48 artículos que se refieren a la resistencia a drogas antimaláricas en Colombia. La literatura revisada está comprendida entre los años 1961 y 1986 (Figura No. 1).

\section{RESULTADOS}

De los 48 artículos, 12 son estudios sobre resistencia realizados en Colombia (in vivo, in vitro, o ambos); del resto, algunos se refieren al tema, pero existen varios que no dicen nada sobre el mismo, aparecían citados como referencia al respecto; inclusive existen
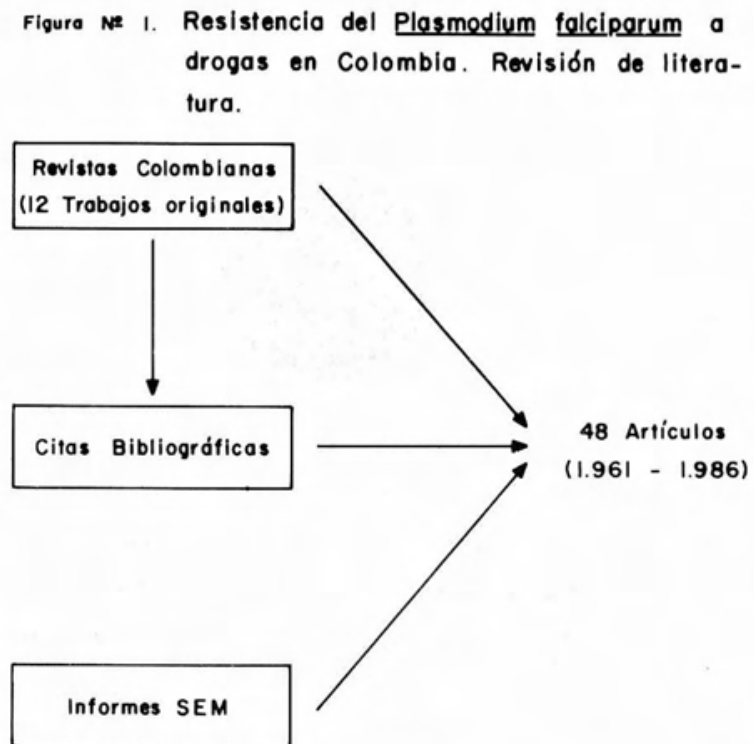

*Profesora del Departamento de Microbiología y Parasitología, Facultad de Medicina, Universidad de Antioquia. MedellínColombia. 
Figura № 2. Características de los Articulos

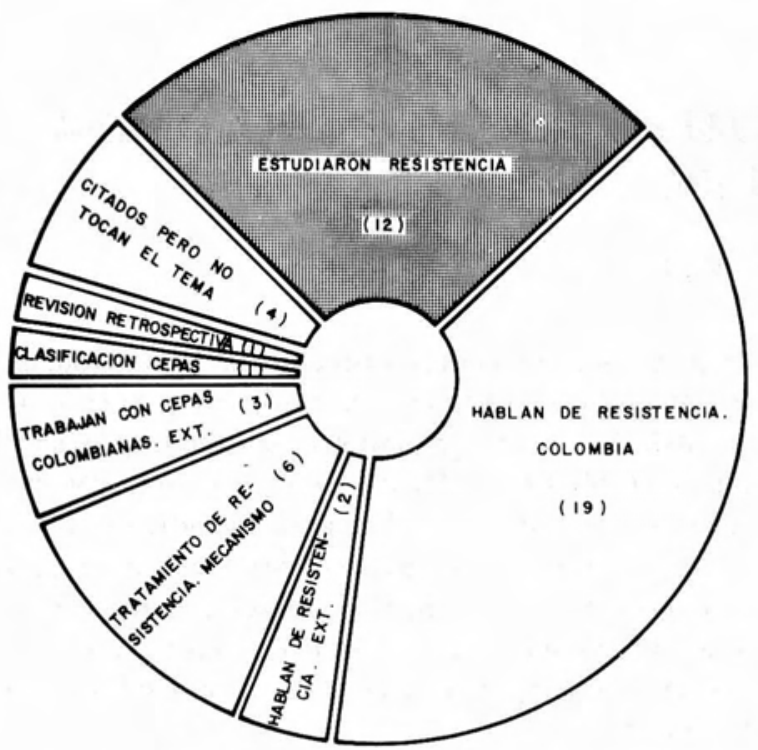

artículos sobre resistencia en otros países que, retomando las bibliografías repetidamente citadas, hacen mención a la resistencia en Colombia (Figura No. 2).

Al totalizar los pacientes estudiados en los 12 trabajos sobre la resistencia en Colombia (in vivo, in vitro, o ambos) $(1,3,4,6,8,20,41,42,43,45,47,48)$ se encontraron 3.087 personas, de las cuales 593 $(19,2 \%)$ se informaron como resistentes, correspondiendo $405(68,3 \%)$ a pruebas in vivo; $91(15,3 \%)$ a pruebas in vitro y $97(16,4 \%)$ a ambas pruebas. Las drogas sometidas a pruebas fueron: amodiaquina, clindamicina, clindamicina-amodiaquina, clindamicinamefloquina, cloroquina, mefloquina-sulfa-pirimetamina y sulfa-pirimetamina (Figura No. 3-a).

La tabla No. 1 resume la información encontrada sobre los 3.087 pacientes y para las drogas probadas.

La resistencia a cloroquina por $P$. falciparum fue estudiada en un total de 2.605 pacientes, de los cuales $516(19,1 \%)$ resultaron resistentes (Figura No. 3-b).

Flgura № 3

a) Resistencia del Plasmodium falciparum a las diferentes Drogas en Co lombia. $\quad 1.961-1.986$.

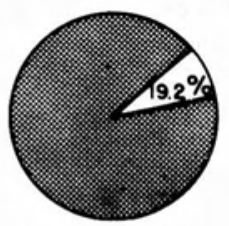

Estudiadas
Resistentes
$\%$ Resistente
In Vivo
In Vitro
In Vivo e In Vitro

b) Resistencia del Plasmodium falciparum a Cloroquina en Colombia. $1.961-1.986$

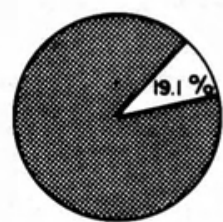

$\begin{array}{lc}\text { Estudiados } & 2.605^{*} \\ \text { Resistentes } & 516 \\ \text { In Vivo } & 334 \\ \text { In Vitro } & 85 \\ \text { In Vivo e In Vitro } & 97 \\ \% \text { Resistencia } & 19.1 \% \\ \text { Grado de Resistencia } & \\ \text { I } & 97 \\ \text { II } & 37 \\ \text { III } & 7 \\ (90 \%) \text { - pociontos fuoron motudlodos } & 1.961\end{array}$


RESISTENCIA DEL P. FALCIPARUM A DROGAS EN COLOMBIA 1961-1986

TABLA № ।

Estudios de "Resistencia" a Drogas en Colombia

$1.961-1.966$

\begin{tabular}{|c|c|c|c|c|c|c|c|}
\hline & Cloroquina & Amodioquino & Sulfa-Pir & Mefloquina & $\mathrm{Cli}+\mathrm{Amo}$ & $\mathrm{Cli}+\mathrm{Q}$ & Total \\
\hline Estudiados & 2.637 & 162 & 64 & 31 & 26 & 31 & * 3.087 \\
\hline Resistentes & $\begin{array}{c}516 \\
(19.5 \%)\end{array}$ & $\begin{array}{c}46 \\
(28.3 \%)\end{array}$ & $\begin{array}{c}22 \\
(34.7 \%)\end{array}$ & $\begin{array}{c}6 \\
(19.3 \%)\end{array}$ & $\begin{array}{c}1 \\
(3.8 \%)\end{array}$ & $\begin{array}{c}2 \\
(6.4 \%)\end{array}$ & 593 \\
\hline In Vivo & 334 & 46 & 22 & - & 1 & 2 & 405 \\
\hline In Vitro & 85 & - & - & 6 & - & - & 91 \\
\hline Dos Métodos & 97 & - & - & - & - & - & 97 \\
\hline
\end{tabular}

Incluye 85 personas estudiadas para Quinina sin Resistencia y 51 personas estudiadas para Mefloquina-Sulfas.

Figura N2 4. Porcentaje de "Resistencia" a las diferentes drogas.

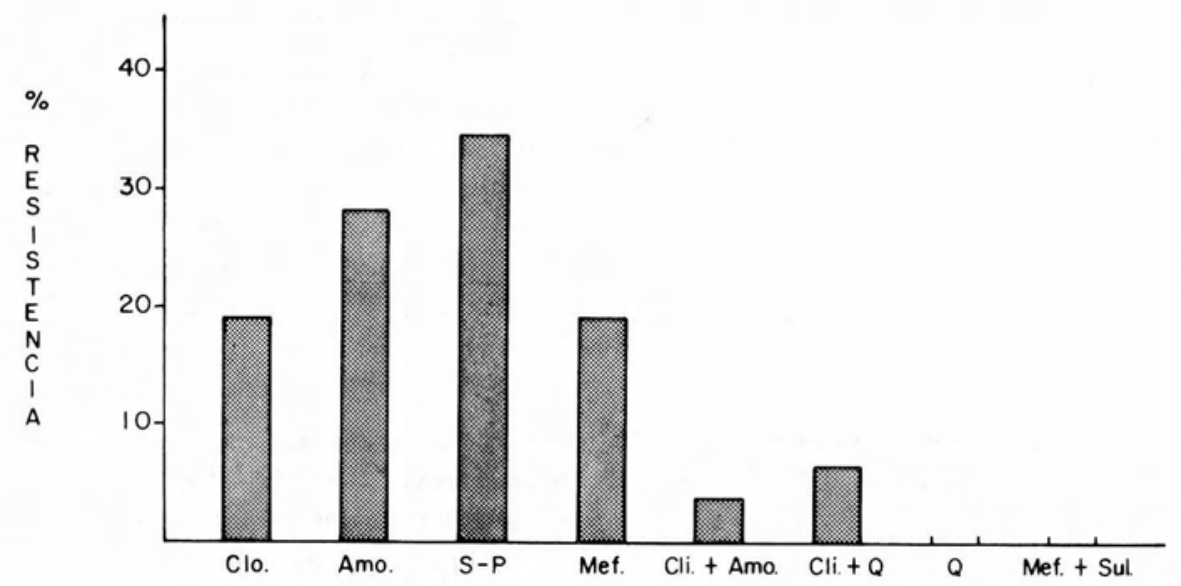

DROGA 


\section{DISCUSION}

La revisión de la bibliografía sobre resistencia surge de la necesidad de conocer la magnitud real de la misma.

La experiencia obtenida en el Laboratorio de Hemoparásitos de la Facultad de Medicina de la Universidad de Antioquia, arroja cifras que no sólo no coinciden con las encontradas en los documentos y trabajos sobre el tema, sino que interroga la magnitud del problema que día a día se presenta a los congresos y reuniones científicas.

Durante 6 años consecutivos el Laboratorio ha atendido a pacientes maláricos procedentes no sólo de Antioquia, sino de todas las zonas maláricas del país.

El esquema de tratamiento utilizado es cloroquinaprimaquina y si bien no se mide exactamente resistencia se controla la respuesta clínica y parasitológica los días 3, 7, 14 y 21. Hasta el momento y con base en resultados obtenidos se puede afirmar que el $88 \%$ de los pacientes presentan al menos 1 control negativo y el $54 \%$ entre 2 y 3 controles negativos.

Lo anterior indica que aproximadamente entre el $70-80 \%$ de los pacientes no complicados responden al tratamiento convencional cloroquina + primaquina.

Solo se establece tratamiento diferencial para las complicaciones y las altas parasitemias.

Ahora bien, según el SEM y para el período enerodiciembre de 1986 (4A) de 41.837 localidades maláricas, sólo se tiene información de 5.048, es decir, su cobertura es de un $12 \%$ con los siguientes resultados: de 477.503 láminas examinadas, 89.251 fueron positivas lo que multiplicado por un factor de corrección de 8 arrojaría cifras de 714.008 personas enfermas por año.

En el caso del $P$. falciparum y sin ningún factor de corrección se informan para el mismo año 30.235 casos.

Ante la magnitud de dichas cifras y cuando la revisión de la totalidad de la literatura colombiana sobre estudios de resistencia, da cuenta de apenas 3.086 casos estudiados entre 1961 y 1986, de los cuales sólo 593 han sido resistentes $(19,2 \%)$ de la totalidad de casos estudiados, no es posible con dicha representatividad plantear conclusiones al respecto. Basta mirar el caso concreto de la cloroquina donde para el mismo período considerado 1961-1986, de los 2.605 casos estudiados, el 90\% (2.948) fueron realizados en 1961. Cómo explicar la variación de la resistencia?

Si a esto se le suman los factores que inciden en el fenómeno y que no han sido considerados como el sitio donde se midió la resistencia, la calidad del trabajo realizado, la correlación in vivo e in vitro de dichas pruebas, es posible que los 593 casos informados como resistentes disminuyan considerablemente.

Las conclusiones -a mi juicio apresuradas- a que han llegado los médicos frente a pacientes maláricos que no presentan mejoría al cuarto día de tratamiento asumiéndolos como resistentes a la droga, han desconocido factores importantes a tener en cuenta en la no respuesta del paciente a dichas drogas. Factores tales como la relación peso corporal-cantidad de droga; situaciones que impiden la absorción total de la droga como el vómito y la diarrea; dosis insuficientes frente al grado de parasitemia o complicaciones; administración de drogas con menos cantidad de principio activo que el anunciado; regreso del paciente a zona endémica con presentación de reinfección; tratamientos que no consultan la diferencia entre pacientes semi-inmunes y no-inmunes, etc.

Inexplicablemente y desconociendo realidades como las aquí planteadas se ha llegado a concluir "que la resistencia del $P$. falciparum a las drogas no sólo existe sino que va progresivamente creciendo hasta llegar a ser semejante a la distribución del $P$. falciparum" (2) o que "la resistencia en Colombia es del 70-90\%" (10) ó más grave aún que esta resistencia es papel causal en la erradicación de la malaria en el país. (12-13).

Estas experiencias, la ausencia de referencias de trabajo concretas y la falta de rigurosidad científica de algunos de los estudios sobre el tema, permiten afirmar que la resistencia a las drogas en Colombia es desconocida haciendo necesarios estudios científicos del problema para medir su prevalencia, evitando el efecto paralizador de dichas conclusiones. 


\section{CONCLUSIONES GENERALES}

1. No se conoce prevalencia real de resistencia a drogas en Colombia.

2. No se conoce la variación de la resistencia a drogas entre 1961-1986 en Colombia.

3. El mayor número de personas estudiadas a resistencia a drogas fue en 1961.

4. Un gran número de trabajos lo que evalúan es respuesta a drogas y no propiamente resistencia.

5. Se dan cifras de resistencia a drogas en Colombia sin mencionar trabajos que las respaldan $(2,21$, $25,28,30,35)$.

6. Algunos estudios parten de la premisa de estar trabajando con pacientes cloroquino resistentes y no se cita cómo se midió esa resistencia.

7. Muchos trabajos dan cifras de resistencia a drogas en Colombia y aún las regiones cloroquino resistentes por información personal o "datos aún no publicados".

8. Algunos trabajos evaluaron respuesta a drogas haciendo controles después del día 14 sin sacar al paciente de zona endémica.

9. Se nota mejor calidad en los estudios realizados en los dos últimos años. No sólo por el número de pacientes, como por la parte técnica en la aplicación del concepto de resistencia tanto in vivo como in vitro.

\section{BIBLIOGRAFIA}

1. Cárdenas H. Estudio sobre distribución geográfica de las cepas de $P$. falciparum resistente a la cloroquina en Colombia, Sur América. Antioquia Médica 1965; 15:329-32.

2. Areas con P. falciparumresitente a la cloroquina. Carta Médica $1980 ; 21: 8$.

3. Restrepo A. Tratamiento con clindamicina de la malaria por P. falciparum resitente. Acta Méd Col 1984; 9:15-21.

4. Restrepo A, Alvarez L. Estudioin vivo de la resitencia del $P$. falciparum a la cloroquina en Colombia. Descripción de la resistencia III. Acta Méd Col 1980; 5:367-379.
4A. Servicio Nacional de Erradicación de la Malaria SEM. Cuestionario sobre el programa de malaria. Enero-diciembre 1986. 1987, abril.

5. Espinal CA y cols. Aislamiento y caracterización del cepas colombianas de Plasmodium falciparum. Biomédica 1982; 3.

6. Espinal CA, Uribe LM. Resistencia del P. falciparum a la combinación sulfa-pirimetamina. Descripción de los primeros tres casos en Colombia. Biomédica 1981; 1.

7. Espinal CA y cols. Tratamiento de las infecciones maláricas producidas por $P$. falciparum y $P$. vivax. Biomédica $1981 ; 1$.

8. Restrepo A, Guzmán J. Tratamiento del P. falciparum con quinina - pirimetamina - sulfas. Acta Méd Col 1982; 7:121125.

9. Restrepo M, Campuzano G. Quimioterapia de la malaria. Antioquia Médica 1976; 26.

10. Espinal CA. Experiencia colombiana en malaria. Foro Malaria Roche nov. 28, 1984.

11. Espinal CA. Conceptos modernos en la respuesta inmune. Mimeografiado INS.

12. Espinal CA, Toro G. Malaria. Mimeografiado.

13. Espinal CA, Eslava A. En ocho años Colombia producirá vacuna antimalárica. Consulta 1981.

14. Restrepo A. Resurgimiento de formas graves de paludismo en Colombia. Antioquia Médica 1974; 24:233-236.

15. Malaria. Resurgimiento resistencia en investigación. N Engl J Med 1983; 308:875-878.

16. Walker Aj, López-Antuñano FJ. Response to drugs of South American strains of Plasmodium falciparum. Transe Royal Soc Trop Med Hyg 1968; 62:654-667.

17. Donald M, Lanier JE. Observations on two Plasmodium falciparum infections with and abnormal response to Chloroquine. Am J Trop Med Hyg 1961; 10:5-9.

18. Martin DJ, Donald M. Chloroquine resistance in P. falciparum. Am J Trop Med Hyg 1961; 10:317-320.

19. Díaz O. El problema malárico en Colombia. Medicina UIS 1969; 2:13-35.

20. Toro G. Malaria: patología clínica. Relatos Médicos 1974; 3 .

21. Mollaret P. Lo trágico del impasse actual del paludismo. Nouv Press Med 1981; 3:61-66.

22. Heineman HS. Qué se debe saber acerca de la malaria. 1975: 2 . 
SYLVIA BLAIR TRUJILLO

23. Escobar ID y col. Malaria: estudio de 55 casos. Medicina UPB 1983; 11 .

24. Ferro C. Paludismo: problema. Salud Pública 1974; 1.

25. Valler A, Bidwell D. Estudio seroepidemiológico de malaria en Colombia. Antioquia Médica 1976; 26.

26. Epidemiología y control de la malaria causada por $P$. falciparum en las Américas. Washington, D.C.: Organización Panamericana de la Salud, 1984; Publicación Científica No. 471.

27. Ferro CA y col. Quimioterapia del paludismo. Tribuna Médica 1979; 59.

28. Grupo científico OMS. Progresos en la quimioterapia del paludismo. Ginebra: Organización Mundial de la Salud, 1984; Serie de Informes Técnicos 711 .

29. Torres H. Estado actual de la malaria. Salud Uninorte 1984; 1:207.

30. Wernsdorfer W. Necesidad de medidas urgentes para combatir el paludismo resistente a los medicamentos. Crónicas de la OMS 1983; 37:12-15.

31. Orientaciones sobre quimioterapia de la malaria. Washington, D.C.: Organización Panamericana de la Salud, 1979; Publicación Científica No. 373.

32. Tratamiento preventivo de la malaria. Drug and therapeutics Vol.20. No. 24-26 y Vol. 21 No. 1-2.

33. Vásquez F. Quimioterapia en áreas con transmisión malárica persistente y resistencia del $P$. falciparum a cloroquina en Panamá.

34. Rodríguez J. Nuestra realidad malárica. Médico Moderno 1979.

35. Rochels P y col. Estudio epidemiológico sobre malaria en la ciudad de Medellín. Salud Pública 1977; 3:3-5.

36. Rodríguez C. Casos de malaria por Plasmodium falciparum resistente ao tratamento pela cloroquina. Arq Hig Saúde Púb 1961; 26:231-235.
37. Rieckman K, López FJ. Choroquine resistance of Plasmodium falciparum in Brazil selected by a simplein vitro method. Bulletin WHO 1971; 45:157-167.

38. López-Antuñano FJ, Wernsdorferw W.In vitro response of chloroquine-resistant Plasmodium falciparum to mefloquine. Bulletin WHO 1979; 57:663-665.

39. Restrepo A, Gutiérrez E. The frequency of glucose 6 phosphate dehydrogenase deficiency in Colombia. Am J Hum Gen $1968 ; 20: 82$.

40. Espinal CA,et al . Sensitivity of Plasmodium falciparum to antimalarial drugs in Colombia. Am J Trop Med Hyg 1985; 34:675.

41. Botero D,et al. Prospective double blind trial of two different doses of mefloquine plus pyrimetamine-sulfa-doxine compared with pyrimetamine-sulfadoxine alone in the treatment of P. falciparum malaria. Bull WHO 1985; 63:731.

42. Espinal CA,et al. Evaluación de clindaminica en combinación con amodiaquina o quinina en infecciones por $P$. falciparum resistente a cloroquina. Acta Med Col 1986; 11.

43. Conner RD,et al. Chloroquine resistance in Plasmodium falciparum malaria on the Pacific Coast of Colombia. Am J Trop Med Hyg 1968; 17:795-799.

44. Arias A, et al. In vitro and in vivo susceptibilities of $P$. falciparum to antimalarial drugs in some regions of Colombia. Acta Méd Col 1982; 7:385.

45. Flórez D. Comportamiento del P. falciparum a 4 aminoquinolinas (Barrancabermeja y Buenaventura 1981-1982). SEM 1982.

46. Flórez D. Estudio de susceptibilidad del $P$. falciparum a drogas en Colombia: Tumaco, Caucasia, Guajira, San José del Guaviare, Barrancambermeja, Buenaventura. (1981-1982). 1982.

47. Enfoque actual sobre el problema de la malaria. Boletín Epidemiológico de Antioquia SSSA 1985; 3. 